\title{
Skin Permeation Enhancement of Ascorbyl Palmitate by Liposomal Hydrogel (Lipogel) Formulation and Electrical Assistance
}

\author{
Sangkil Lee, Jaehwi LeE, * and Young Wook CHOI* \\ Division of Pharmaceutical Sciences, College of Pharmacy, Chung-Ang University; 221 Heuksuk-dong, Dongjak-gu, Seoul \\ 156-756, Korea. $\quad$ Received July 13, 2006; accepted November 20, 2006
}

\begin{abstract}
To enhance skin permeation of ascorbyl palmitate (AsP), it was encapsulated in liposomes, and formulated into liposomal hydrogel (lipogel) by dispersing the liposome into poloxamer hydrogel matrix. To improve the skin permeation of AsP, we applied electric current supplying system that mimics an electric skin massager. We evaluated the effects of composition and surface charge of the liposomes and electrical assistance on the skin permeation of AsP. In the passive transport study, the permeated amounts of AsP from all the lipogels tested were higher than that of control hydrogel which contains Transcutol ${ }^{\circledR}$ used to solubilize AsP. In the cathodal delivery condition with a fixed cathodal current of $0.4 \mathrm{~mA} / \mathrm{cm}^{2}$, the skin permeation characteristics of the negative lipogels were superior to that obtained with the neutral lipogels and the drug permeation was more increased with increased surface negative charge of the liposomes. In conclusion, the lipogel system was thought as a helpful drug delivery system to enhance skin permeation of AsP. Combined use of negative lipogel with cathodal electric assistance was found to be promising in enhancing the skin delivery of AsP.
\end{abstract}

Key words skin permeation; liposome; lipogel; electrical assistance; ascorbyl palmitate

It was widely reported that liposomes have been used in delivery of active compounds into the skin. Liposomes have been considered to serve as 'organic solvent' for the solubilization of poorly soluble drugs and local depot for the dermally active compounds. Furthermore, the potential applicability of liposomes as a topical drug delivery system has been reported since Mezei and Gulasekharam had demonstrated first that liposomes facilitate the accumulation of the drug within the epidermis and dermis. ${ }^{1,2}$ They may also serve as a skin permeation enhancer and promote dermal delivery leading to greater dermal localization of drugs. ${ }^{3)}$

The major advantages of topical liposome formulations include the followings: i) reduced side effects and incompatibility that may arise from undesirably high systemic absorption of drugs, ii) enhanced accumulation of drugs at the site of administration as a result of the high affinity of liposomes with stratum corneum, and iii) an ability to incorporate a wide variety of hydrophilic and hydrophobic drugs. $\left.{ }^{4}\right)$

To increase skin permeation of drugs, electrical assistance such as iontophoresis and electroporation has been examined which are aimed at overcoming the low drug permeation caused mainly by stratum corneum. ${ }^{5}$ In other papers, the combined use of iontophoresis and liposomal formulation in transdermal drug delivery was reported. It was expected that while liposomes may act as a drug reservoir to continuously release drug into the skin surface, iontophoresis may pump the released drug into the dermis via electrical repulsive force. ${ }^{6,7)}$

Recently, people use electric skin massage devices for aesthetic purposes via delivery of skin whitening or anti-aging bioactive materials. However, there has been little study regarding the impact of electric skin massager on skin delivery efficiency of the bioactive materials. The electric supplying condition of the massage devices is different from iontophoresis. For an iontophoresis, generally continuous or pulsatile electric current is supplied at the fixed position but the electric current supplying position of skin massage devices is very irregular because the electric current donating site is continuously changed randomly.
There has been a report concerning formulation of ascorbic acid (AsA) employing liposome, multiple emulsion and cream but the hydrophilic nature of AsA makes it difficult to be encapsulated with a high yield into the delivery systems. ${ }^{8)}$ For this reason, ascorbyl palmitate (AsP), a prodrug of ascorbic acid was introduced as an alternative skin whitening and anti-aging agent. AsP has been shown to overcome main drawbacks associated with AsA such as instability to light, oxygen and heavy metals, and the hydrophobic nature of AsP makes it possible to be efficiently encapsulated into topical drug delivery systems with high yields. ${ }^{9)}$

In this study, we prepared AsP-containing liposomes and formulated into a liposomal hydrogel (lipogel) by dispersing the liposome solution into poloxamer hydrogel matrix. We, then, evaluated the skin permeability of AsP in lipogels and also the permeation enhancing effect of electrical assistance by employing electric skin massage condition at a fixed current of $0.4 \mathrm{~mA} / \mathrm{cm}^{2}$. Basically, the goal of the study must be an evaluation of the accumulation of ascorbyl palmitate in the skin. However, our previous study showed that ascorbyl palmitate, after being permeated, was rapidly hydrolyzed to its parent drug, ascorbic acid but, since the stability of ascorbic acid in the skin tissue was so poor $\left(t_{90 \%}<1.0 \mathrm{~h}\right)$, it was practically impossible to measure the skin accumulation of ascorbic acid. Thus, we considered that the skin permeation of ascorbyl palmitate can be measured as a tool to compare the ability of delivering ascorbyl palmitate into the skin because if a drug is to be accumulated in the skin it must first be transported into the skin.

\section{MATERIALS AND METHODS}

Materials Ascorbyl palmitate (AsP) was provided from Takeda Co., Ltd. (Chuo-ku, Osaka, Japan). Dimyristoylphosphatydilcholine (DMPC) and dicetylphosphate (DCP) were purchased from Sigma Chemical Co., Ltd. (St. Louis, MO, U.S.A.). Poloxamer 407 was obtained from BASF Korea (Chung-gu, Seoul, Korea) and all other solvents and chemicals were of reagent grade and used as received. 
Preparation of Liposomes Multilamellar vesicles containing AsP were prepared by traditional hydration method. ${ }^{10)}$ Briefly, neutral liposomes were prepared with only DMPC and negatively charged liposomes (negative liposomes) were prepared with DMPC and DCP (10,20\%). The lipid $(67.79 \mathrm{mg})$ and AsP $(50 \mathrm{mg})$ were mixed and dissolved in enough volume of chloroform. The thin lipid film was obtained by removing the organic solvent under a vacuum condition using a rotary evaporator (Eyela, Tokyo Rikakikai Co., Japan). The lipid film was incubated for $6 \mathrm{~h}$ at room temperature and rehydrated with $5 \mathrm{ml}$ of $\mathrm{pH} 7.0$ PBS.

Measurement of Liposome Size and Size Distribution The diameter of the liposomes was determined using dynamic light scattering system (LPA PARIII, Otsuka Electronics, Japan) at $25^{\circ} \mathrm{C}$ at conditions of scattering angle of $90^{\circ}$ and 12000 count per second.

Measurement of Zeta Potential Liposome dispersions were diluted with $\mathrm{pH}$ 7.4 PBS and zeta potential values were measured at $25^{\circ} \mathrm{C}$ using Zetasizer 2000 (Malvern Instruments, Malvern, U.K.).

Measurement of Encapsulation Efficiency Liposomal encapsulation efficiency was measured by gel filtration method using Sephadex column (Sephadex G-25 column, Amersham Pharmacia, Sweden). The free drug was separated from the liposomes and the content of liposomally encapsulated drug was measured with HPLC. To measure encapsulation efficiency, liposomes were dissolved into 5\% Triton-X 100 solution and the encapsulation ratio was calculated from the ratio of measured amount of drug to total drug added.

Preparation of Control Hydrogels To compare the effects of using liposomes on the skin permeation of AsP, liposome-free control hydrogels were prepared separately. Because of low water solubility of AsP, it was solubilized with Transcutol ${ }^{\circledR}$ and dispersed the mixture into poloxamer gel matrix, in which the amount of AsP added was $0.2 \%$.

Preparation of Liposomal Hydrogels The liposomal hydrogel (lipogel) was prepared by dispersing liposomal solution into pre-swollen $25 \%$ poloxamer gel matrix at $4{ }^{\circ} \mathrm{C}$. The final concentration of AsP in lipogels was the same as the control hydrogel.

Passive Skin Permeation Study Male Sprague-Dawley rats (12-14 weeks old, weighing $220 \pm 10 \mathrm{~g}$ ) were used for passive in vitro skin permeation studies. The dorsal skin was removed carefully leaving the fat tissue and rinsed with PBS. Prepared skin samples were stored at $-70^{\circ} \mathrm{C}$ until skin permeation experiments. One gram of hydrogel samples was loaded onto the donor compartment of Franz diffusion cells with a permeation area of $2.00 \mathrm{~cm}^{2}$ and PBS containing $30 \%$ ethanol was used as a receptor solution $(11 \mathrm{ml})$ maintained at $37 \pm 0.5^{\circ} \mathrm{C}$.

Skin Permeation Study under Electrically Assisted Condition The experimental condition of electrically assisted skin permeation studies was basically the same as the passive diffusion studies except for the application of electric current supplying system. To mimic electric current supplying conditions from skin massager, we prepared power supply and applied a fixed cathodal current of $0.4 \mathrm{~mA} / \mathrm{cm}^{2}$. The reason for being chosen a current of $0.4 \mathrm{~mA} / \mathrm{cm}^{2}$ was because current densities below $0.5 \mathrm{~mA} / \mathrm{cm}^{2}$ were generally considered to be well tolerated in humans. ${ }^{11)}$ In this study, the elec- tric current was applied for $30 \mathrm{~min}$ in a pulsatile manner with on and off every $30 \mathrm{~s}$ to simulate skin massage condition.

HPLC Assay Normal phase $\mu$-Bondapak $\left(\mathrm{Li}-\mathrm{NH}_{2}\right.$, $3.9 \times 300 \mathrm{~mm}, 10 \mu \mathrm{m}$, Waters, U.S.A.) column was used to analyze AsP. The composition of mobile phase was methanol : phosphate buffer $(0.02 \mathrm{M}, \mathrm{pH} 3.5)=70: 30(\mathrm{v} / \mathrm{v})$ with a flow rate of $1.0 \mathrm{ml} / \mathrm{min}$. Detection wavelength was $255 \mathrm{~nm}$ and injection volume was $20 \mu \mathrm{l}$.

Data Analysis In our previous study, it was demonstrated that AsP was hydrolyzed to free ascorbic acid (AsA) by skin esterases (data now shown), the amount of drug permeated was calculated from sum of molar amount of AsP permeated and generated AsA, per unit area $\left(Q_{\mathrm{P}}, \mathrm{mmol} / \mathrm{cm}^{2}\right)$. Flux $\left(J_{\mathrm{s}}\right)$ was calculated using as the following equation.

$$
J_{\mathrm{s}}=\frac{d Q}{d t} \cdot \frac{1}{A}
$$

where, $d Q / d t$ is the slope of the linear portion of the graph and $A$ is the effective diffusion area.

All data are represented as mean \pm S.E. Statistical analysis of data sets for each group of measurements was performed using the Student's $t$-test. The levels of significance were taken as $p<0.05$ except where stated otherwise.

\section{RESULTS AND DISCUSSION}

The size of the neutral liposomes was about $1000 \mathrm{~nm}$ and that of negatively charged liposomes was ranged from 600 to $700 \mathrm{~nm}$ (Table 1). The surface charges of the liposomes containing DCP were $-32.50 \pm 2.22(\mathrm{DMPC}: \mathrm{DCP}=9: 1)$ and $-47.52 \pm 6.72 \mathrm{mV}$ (DMPC : $\mathrm{DCP}=8: 2$ ), respectively and absolute value of the surface charges increased when the amount of DCP added increased.

Before starting skin permeation study, we formulated control hydrogel with Transcutol ${ }^{\circledR}$ to compare it with the liposome system. We chose Transcutol ${ }^{\circledR}$ since it can solubilize AsP in the control hydrogel.

In the passive skin transport study, the permeated amount $\left(Q_{\mathrm{P}}\right)$ and flux value $\left(J_{\mathrm{s}}\right)$ of AsP in the lipogels were significantly higher than those obtained with the control hydrogels (Fig. 1, Table 2). And, $Q_{\mathrm{P}}$ and $J_{\mathrm{s}}$ obtained from neutral lipogels were slightly higher than those of negative lipogels in spite of larger vesicular size of the neutral liposomes.

There have been controversies regarding skin permeation characteristics of liposomes such as whether they increase or decrease skin permeation of encapsulated drugs. In one

Table 1. Physical Characteristics of Neutral and Negatively Charged Liposomes Containing Acorbyl Palmitate

\begin{tabular}{cccc}
\hline \hline $\begin{array}{c}\text { Composition } \\
\text { of } \\
\text { liposomes }\end{array}$ & $\begin{array}{c}\text { Particle } \\
\text { size } \\
(\mathrm{nm})\end{array}$ & $\begin{array}{c}\text { Zeta } \\
\text { potential } \\
(\mathrm{mV})\end{array}$ & $\begin{array}{c}\text { Encapsulation } \\
\text { efficiency } \\
(\%)\end{array}$ \\
\hline Neutral & $1027.4 \pm 99.9$ & ND & $90.5 \pm 0.6$ \\
Negative & $611.8 \pm 16.0$ & $-32.5 \pm 2.2$ & $99.5 \pm 0.0$ \\
$\begin{array}{c}\text { DMPC : DCP } \\
(9: 1)\end{array}$ & & & \\
Negative & $650.1 \pm 22.4$ & $-47.5 \pm 6.7$ & $76.1 \pm 0.9$ \\
$\begin{array}{c}\text { DMPC:DCP } \\
(8: 2)\end{array}$ & & & \\
\hline
\end{tabular}

ND: Not determined. Data are expressed as mean \pm S.D., $n=3$. 


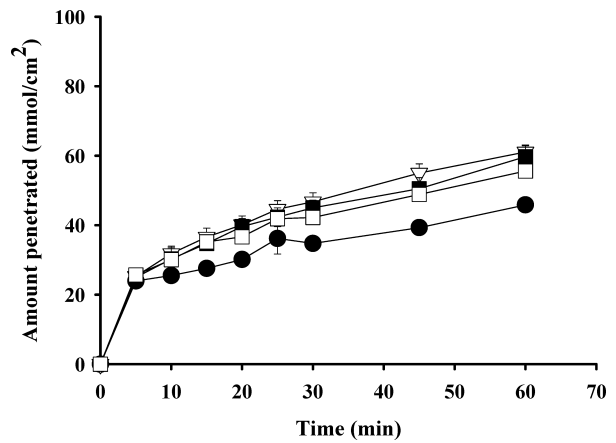

Fig. 1. In Vitro Passive Ascorbyl Palmitate Transport Profiles from Various Formulations

Each data point indicates mean \pm S.E. $(n=3)$. Key: $\bullet$, control hydrogel (Transcutol ${ }^{\circledR}$ $5 \%$ ); $\nabla$, neutral lipogel; $\mathbf{\square}, 10 \%$ DCP negative lipogel (DMPC : $\mathrm{DCP}=9: 1$ ); $\square, 20 \%$ DCP negative lipogel (DMPC : $\mathrm{DCP}=8: 2$ ).

Table 2. Skin Permeation Parameters of Ascorbyl Palmitate in Lipogels Prepared with Neutral and Negatively Charged Liposomes after $1 \mathrm{~h}$

\begin{tabular}{|c|c|c|c|}
\hline & $\begin{array}{l}\text { Liposome } \\
\text { composition }\end{array}$ & $\begin{array}{c}Q_{\mathrm{P}} \\
\left(\mathrm{mmol} / \mathrm{cm}^{2}\right)\end{array}$ & $\begin{array}{c}J_{\mathrm{s}} \\
\left(\mathrm{mmol} / \mathrm{cm}^{2} / \mathrm{h}\right)\end{array}$ \\
\hline \multirow[t]{6}{*}{ Passive transport } & Control hydrogel & $45.8 \pm 0.7$ & $23.9 \pm 1.2$ \\
\hline & Neutral lipogel** & $61.1 \pm 2.5$ & $39.1 \pm 1.8$ \\
\hline & Negative & $59.7 \pm 3.4$ & $37.9 \pm 3.3$ \\
\hline & $\begin{array}{c}\text { DMPC : DCP } \\
(9: 1)^{* *}\end{array}$ & & \\
\hline & Negative & $55.6 \pm 0.6$ & $32.6 \pm 1.5$ \\
\hline & $\begin{array}{c}\text { DMPC : DCP } \\
(8: 2)^{*, \dagger}\end{array}$ & & \\
\hline \multirow{5}{*}{$\begin{array}{c}\text { Electrically } \\
\text { assisted } \\
\text { transport }\end{array}$} & Neutral ${ }^{* *,+}$ & $75.2 \pm 1.8$ & $44.9 \pm 2.0$ \\
\hline & Negative & $76.0 \pm 4.1$ & $40.3 \pm 3.8$ \\
\hline & $\begin{array}{c}\text { DMPC : DCP } \\
(9: 1)^{* * * *}\end{array}$ & & \\
\hline & Negative & $86.8 \pm 1.4$ & $43.8 \pm 5.8$ \\
\hline & $\begin{array}{c}\text { DMPC : DCP } \\
(8: 2)^{* *, *}\end{array}$ & & \\
\hline
\end{tabular}

$* Q_{\mathrm{P}}$ and $J_{\mathrm{s}}$ values are significantly different from control hydrogel $(p<0.05) . * * Q_{\mathrm{P}}$ and $J_{\mathrm{s}}$ values are significantly different from control hydrogel $(p<0.01),{ }^{\dagger} Q_{\mathrm{P}}$ value is significantly different from neutral lipogel. Comparison was performed only in passive transport condition $(p<0.05) . \ddagger Q_{\mathrm{P}}$ value is significantly different from passive delivery condition $(p<0.05)$. Comparison was performed between same liposomal compositions.

paper, it was reported that liposomes decrease the flux of loaded drugs as phospholipids may form an extra lipid barrier onto the skin surface. ${ }^{12)}$ However, many reports have revealed that an increase in skin localization and flux by phosphatidylcholine was prominent. In those studies, they suggested that the permeation enhancing effect shown by liposomes might predominantly be induced by the action of phosphatidylcholine ingressing into the lipid domain of the stratum corneum. Moreover after pre-treatment of the skin with phospholipids, the skin permeation rate of drug was also enhanced. From these results, it was considered that phopholipids may affect lipid domain of the stratum corneum, leading to the increased fluidity and thereby decreased barrier property of the stratum corneum. ${ }^{13)}$

There are several factors affecting skin permeation of liposomes such as particle size, composition and surface charge. Generally, vesicular size has been known to be one of the most important factors in the skin permeation of liposome, but it was demonstrated that vesicular size over $200 \mathrm{~nm}$ did not significantly affect skin permeation of drugs. ${ }^{14)}$ In our

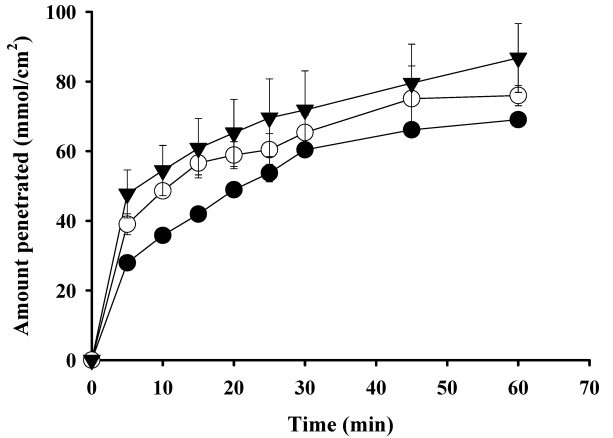

Fig. 2. In Vitro Skin Permeation Profile of Ascorbyl Palmitate by Cathodal Transport

Each data point indicates mean \pm S.E. $(n=3)$. Key: $\bullet$, neutral lipogel; $\bigcirc, 10 \%$ DCP negative lipogel (DMPC: $\mathrm{DCP}=9: 1$ ); $\mathbf{\nabla}, 20 \%$ DCP negative lipogel (DMPC : $\mathrm{DCP}=$ $8: 2$ ).

passive permeation study, the size of all liposomes tested was larger than $200 \mathrm{~nm}$, it was thus, expected that liposome itself does not have an effect on drug permeation. The $Q_{\mathrm{P}}$ values obtained from neutral lipogels $\left(61.08 \pm 2.48 \mathrm{mmol} / \mathrm{cm}^{2}\right)$ were slightly greater than those obtained with negative lipogels $\left(59.73 \pm 3.37,55.58 \pm 0.59 \mathrm{mmol} / \mathrm{cm}^{2}\right.$ for lipogels containing liposomes of DMPC:DCP ratio of $9: 1,8: 2$, respectively). The $Q_{\mathrm{P}}$ values of negative lipogel were decreased with increasing amount of DCP. The skin can act like an ion-exchange membrane, as a result, negatively charged molecules may have difficulty to traverse the membrane than neutral or positively charged molecules. ${ }^{15)}$ Actually, the permeation of AsP encapsulated in negative lipogels was reduced and there was a significant difference $(p<0.05)$ in the permeation rates between neutral lipogel and negative lipogel containing $20 \%$ DCP in passive transport study. The skin permeation of AsP from neutral lipogel was the greatest proportion inspite of larger vesicular size of neutral liposomes than the lipogels containing negatively charged liposomes. This result showed one aspect of permselectivity of skin, so-called 'Donnan exclusion effect', ${ }^{15)}$ which explains the skin permeation characteristics of charged molecules.

As shown in Fig. 2, the skin permeation of AsP from all lipogel formulations was further enhanced by cathodal electric supply of $0.4 \mathrm{~mA} / \mathrm{cm}^{2}$, compared to the passive delivery condition $(p<0.05)$ and the skin permeability of AsP increased with increasing surface negative charges. As mentioned above, liposomal formulations may enhance the skin permeation of drugs by interacting with lipid bilayers. However, from the passive and cathodal transport studies, we noticed that the skin permeation of AsP in the liposomal gel systems was also associated with the liposomal composition.

In the passive delivery, the amount of AsP permeated for initial $5 \mathrm{~min}\left(Q_{\mathrm{P}, 5 \mathrm{~min}}\right)$ was not considerably different from each other whether the surface of liposomes were charged or not (Fig. 1). However, after $1 \mathrm{~h}$, the amount and rate of skin penetration of AsP were in an order of neutral liposome $>10 \%$ DCP liposome $>20 \%$ DCP liposome $>$ control. In case of the skin permeation for $5 \mathrm{~min}$, the reason that practically no difference in skin permeation was observed was probably because a period of $5 \mathrm{~min}$ was too short for the skin to demonstrate ion-exclusion property.

However, there were significant differences between neutral and negative lipogels under the electrically assisted con- 


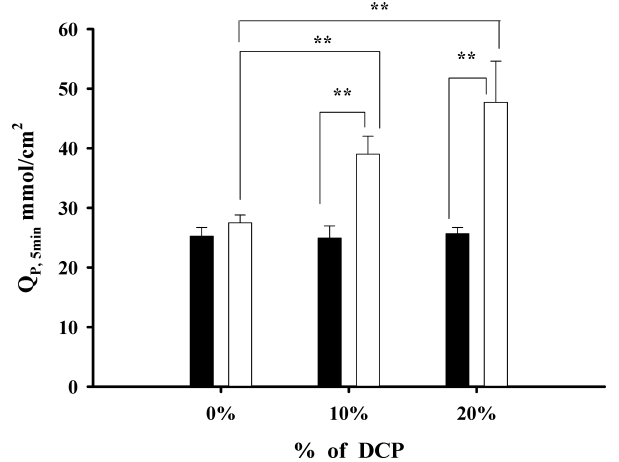

Fig. 3. Comparison for $Q_{\mathrm{P}, 5 \min }$ by Passive and Electrically Assisted Condition

Each data point is mean \pm S.E. $(n=3)$. Key: $\mathbf{\square}$, passive delivery; $\square$, cathodal delivery. $* *$ Significantly different from each other $(p<0.01)$

dition. As shown in Figs. 2 and 3, the $Q_{\mathrm{P}, 5 \text { min }}$ was increased about $1.6(10 \%$ DCP) and $1.9(20 \%$ DCP) times by cathodal electric supply condition $(p<0.01)$. Although the pores in the skin tissue occupy only $0.1 \%$ of the total area of the skin, it is known that the major absorption route of electrically assisted transport may be appendageal pores including sweat ducts and hair follicles. ${ }^{16)}$ When considering this, the AsP encapsulated in negatively charged liposomes could easily be transported through the appendageal pores by electrical assistance. This can certainly be supported by the fact that the $Q_{\mathrm{P}}$ values of negative lipogel were higher than that of neutral lipogel in electrically assisted transport study.

In conclusion, the lipogel systems showed good skin permeation characteristics compared to control hydrogel containing Transcutol ${ }^{\circledR}$ which is known as a drug solubilizer. Furthermore, the cathodal electric assistance increased skin permeation of liposomal AsP by electric repulsion probably via the appendageal routes. The lipogels were found to be promising delivery system of AsP and combination of nega- tively charged lipogel and cathodal electric assistance was able to further enhance the skin penetration of AsP.

Acknowledgements The authors would like to thank for financial support by Korea Institute of Industrial Technology Evaluation and Planning (ITEP) in Korea. Also, this work was supported by the Seoul R\&BD Program.

\section{REFERENCES}

1) Mezei M., Gulasekharam V., Life Sci., 26, 1473-1477 (1980).

2) Mezei M., Gulasekharam V., J. Pharm. Pharmacol., 34, 473-474 (1981).

3) Schreier H., Bouwstra J., J. Control. Release, 30, 1-15 (1994).

4) Weiner N., Lieb L., Niemiec S., Ramachandran C., Hu Z., Egbaria K., J. Drug Target., 2, 405-410 (1994).

5) Banga A. K., "Electrically Assisted Transdermal and Topical Drug Delivery," Chap. 1, Taylor and Francis Ltd., London, 1998.

6) Vutla N. B., Betageri G. V., Banga A. K., J. Pharm. Sci., 85, 5-8 (1996).

7) Fang J. Y., Sung K. C., Lin H. H., Fang C. L., J. Control. Release, 60, $1-10$ (1999).

8) Gallarate M., Carlotti M. E., Trotta M., Bovo S., Int. J. Pharm., 25, 233-241 (1999).

9) Austria R., Sementazo A., Bettero A., J. Pharm. Biomed. Anal., 15, 795-801 (1997)

10) Bangham A. D., Hill M. W., Miller M. G. A., "Preparation and Use of Liposomes as Models of Biological Membranes," ed. by Korn E. D., Plenum Press, New York, 1974, pp. 56-61.

11) Walters K. A., Hadgraft J., "Pharmaceutical Skin Penetration Enhancement," Marcel Dekker, Inc., New York, 1993, pp. 311-333.

12) Kirjavainen M., Urtti A., Koskela R.V., Kiesvaara J., Mökkönen J. M., Eur. J. Pharm. Sci., 7, 279-286 (1999).

13) Fang J. Y., Hong C. T., Chiu W. T., Wang Y. Y., Int. J. Pharm., 219, $61-72$ (2001).

14) Šentjure M., Vrhovnik K., Kristl J., J. Control. Release, 59, 87—97 (1999).

15) Ronald R. B., “Transdermal Drug Delivery," Chap. 11, ed. by Hadgraft J., Guy R. H., Marcel Dekker, New York, 1980, pp. 247-291.

16) Singh P., Maibach H. I., Adv. Drug Deliv. Rev., 18, 379-394 (1996). 